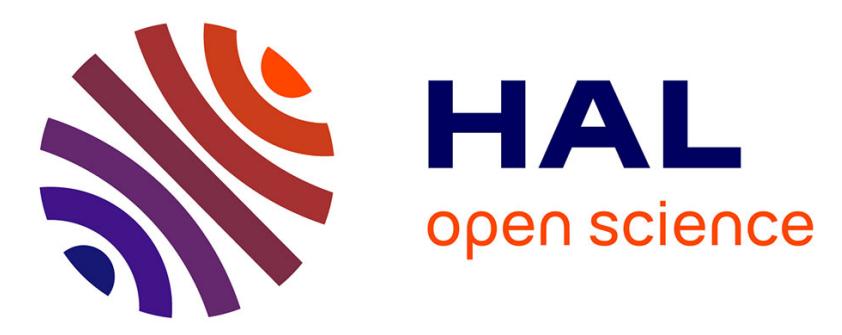

\title{
Effect of the dynamic topology on the performance of PSO-2S algorithm for continuous optimization
}

\author{
Abbas El Dor, Patrick Siarry
}

\section{To cite this version:}

Abbas El Dor, Patrick Siarry. Effect of the dynamic topology on the performance of PSO-2S algorithm for continuous optimization. Machine Learning, Optimization, and Big Data: First International Workshop, MOD 2015, Jul 2015, Taormina, Sicily, Italy. hal-01237446

\section{HAL Id: hal-01237446 \\ https://hal.inria.fr/hal-01237446}

Submitted on 3 Dec 2015

HAL is a multi-disciplinary open access archive for the deposit and dissemination of scientific research documents, whether they are published or not. The documents may come from teaching and research institutions in France or abroad, or from public or private research centers.
L'archive ouverte pluridisciplinaire HAL, est destinée au dépôt et à la diffusion de documents scientifiques de niveau recherche, publiés ou non, émanant des établissements d'enseignement et de recherche français ou étrangers, des laboratoires publics ou privés. 


\title{
Effect of the dynamic topology on the performance of PSO-2S algorithm for continuous optimization
}

\author{
Abbas EL DOR ${ }^{1}$ and Patrick SIARRY ${ }^{2}$ \\ 1 Ecole des Mines de Nantes, TASC INRIA (CNRS UMR 6241), \\ 4 rue Alfred Kastler, 44300 Nantes, FRANCE \\ abbas.eldor@mines-nantes.fr \\ 2 Université de Paris-Est Créteil, LiSSi (E.A. 3956), \\ 122 rue Paul Armangot, 94400 Vitry-sur-Seine, FRANCE \\ siarry@u-pec.fr
}

\begin{abstract}
PSO-2S is a multi-swarm PSO algorithm using charged particles in a partitioned search space for continuous optimization problems. In order to improve the performance of $\mathrm{PSO}-2 \mathrm{~S}$, this paper proposes a novel variant of this algorithm, called DPSO-2S, which uses the Dcluster neighborhood topologies to organize the communication networks between the particles. Experiments were conducted on a set of classical benchmark functions. The obtained results prove the effectiveness of the proposed algorithm.
\end{abstract}

\section{Introduction}

The concept of particle swarm optimization (PSO) is based on social behavior to exchange information between the particles in a swarm. Thus this property can be modelized thanks to a graph: two particles $P_{i}$ and $P_{j}$ of the swarm $S$ are connected if a communication can be established between them. The set of edges between each particle $P_{i}$ and its neighbours $N e_{i}$ forms the communication graph, also called the topology. Hence, the chosen topology can greatly affect the performance of the PSO algorithm. In this paper, we present a new dynamic topology, called Dcluster, which is a combination of two existing topologies (Four-clusters [7] and Wheel [6]). This topology was integrated in our proposed algorithm called PSO-2S, introduced in [2]. PSO-2S is a multi-swarm PSO algorithm using charged particles in a partitioned search space for continuous optimization problems. The performance of PSO-2S with the Dcluster topology is analysed and compared to that of PSO-2S without Dcluster, using a set of benchmark test functions. Comparisons show that the use of Dcluster improves significantly the performance of PSO-2S.

\section{Particle Swarm Optimization}

The particle swarm optimization (PSO) [4] is inspired originally by the social and cognitive behavior existing in the bird flocking. The algorithm is initialized 
with a population of particles randomly distributed in the search space, and each particle is assigned a randomized velocity. Each particle represents a potential solution to the problem. The particles fly over the search space, keeping in memory the best solution encountered. At each iteration, each particle adjusts its velocity vector, based on its momentum, influences of its best solution and of the best solution of its neighbors, then computes a new point to be evaluated. The displacement of a particle is influenced by three components:

1. Physical component: the particle tends to keep its current direction of displacement;

2. Cognitive component: the particle tends to move towards the best site that it has explored until now;

3. Social component: the particle tends to rely on the experience of its congeners, then moves towards the best site already explored by its neighbors.

In this paper, the swarm size is denoted by $s$, and the search space is $n-$ dimensional. In general, a particle $i$ has three attributes: the current position $X_{i}=\left(x_{i, 1}, x_{i, 2}, \ldots, x_{i, n}\right)$, the current velocity vector $V_{i}=\left(v_{i, 1}, v_{i, 2}, \ldots, v_{i, n}\right)$ and the past best position Pbest ${ }_{i}=\left(p_{i, 1}, p_{i, 2}, \ldots, p_{i, n}\right)$. The best position found in the neighborhood of the particle $i$ is denoted by $G_{b e s t}=\left(g_{i, 1}, g_{i, 2}, \ldots, g_{i, n}\right)$. These attributes are used to update iteratively the state of each particle in the swarm. The objective function to be minimized is denoted by $f$. The velocity vector $V_{i}$ of each particle is updated using the best position it visited so far and the overall best position visited by its neighbors. Then, the position of each particle is updated using its updated velocity per iteration. At each step, the velocity of each particle and its new position are updated as follows:

$$
\begin{gathered}
v_{i, j}(t+1)=w v_{i, j}(t)+c_{1} r_{1_{i, j}}(t)\left[p_{i, j}(t)-x_{i, j}(t)\right]+c_{2} r_{2_{i, j}}(t)\left[g_{i, j}(t)-x_{i, j}(t)\right] \\
x_{i, j}(t+1)=x_{i, j}(t)+v_{i, j}(t+1)
\end{gathered}
$$

$x_{i, j}$ is the position and $v_{i, j}$ is the velocity of the $i$ th particle $(i \in 1,2, \ldots, s)$ of the $j$ th dimension $(j \in 1,2, \ldots, n)$. Where $w$ is called inertia weight, $c_{1}, c_{2}$ are the learning factors and $r_{1}, r_{2}$ are two random numbers selected uniformly in the range $[0,1]$.

\section{PSO-2S algorithm}

In this section, we present the first version of PSO-2S [2]. PSO-2S is based on three main ideas. The first is to use two kinds of swarms: a main swarm, denoted by $\mathrm{S} 1$, and $s$ auxiliary ones, denoted by $\mathrm{S} 2{ }_{\mathrm{i}}$, where $1 \leq i \leq s$. The second idea is to partition the search space into several zones in which the auxiliary swarms are initialized (the number of zones is equal to the number of auxiliary swarms, thus is equal to $s$ ). The last idea is to use the concept of the electrostatic repulsion heuristic to diversify the particles for each auxiliary swarm in each zone.

To construct S1, we propose to perform the auxiliary swarms $\mathrm{S} 2_{\mathrm{i}}$ several times in different areas, and then each best particle for each $\mathrm{S}_{2}$ is saved and 
considered as a new particle of S1. To do so, the population of each auxiliary swarm is initialized randomly in different zones (each $S 2_{\mathrm{i}}$ is initialized in its corresponding zone $i$ ). After each of these initializations, $K$ displacements of particles, of each $\mathrm{S} 2_{\mathrm{i}}$, are performed in the same way of standard PSO. Then the best solution found by each auxiliary swarm is added to S1. The number of initializations of $\mathrm{S}_{\mathrm{i}}$ is equal to the number of particles in $\mathrm{S} 1$.

As we mentioned above the second idea is to partition the search space $\left[\min _{d}, \max _{d}\right]^{N}$ into several zones ( $\max _{z o n e}$ zones). Then, we calculate the center $_{d}$ and the step $_{d}$ of each dimension separately, according to (3) and (4). The $s t e p_{d}$ are similar in the case of using a square search space.

$$
\begin{gathered}
\text { center }_{d}=\left(\max _{d}-\min _{d}\right) / 2 \\
\text { step }_{d}=\text { center }_{d} / \max _{z o n e}
\end{gathered}
$$

where $\max _{z o n e}$ is a fixed value, and $d$ is the current dimension $(1 \leq d \leq N)$.

The sizes of the zones of the partitioned search space are different $\left(Z_{1}<\right.$ $\left.Z_{2}<\ldots<Z_{\max _{z o n e}}\right)$. Therefore, the number of particles in $\mathrm{S} 2_{\mathrm{i}}$, denoted by $S 2_{\text {isize }}$, depends on its corresponding zone size. Indeed, a small zone takes less particles and the number of particles increases when the zone becomes larger. The size of each auxiliary swarm is calculated as follows:

$$
S 2_{\text {isize }}=\text { num }_{\text {zone }} * n b_{\text {particle }}
$$

where num $_{z o n e}=1,2, \ldots, \max _{z o n e}$, is the current zone number and $n b_{\text {particle }}$ is a fixed value. After the initializations of the auxiliary swarms in different zones $\left(Z_{i}, \mathrm{~S} 2_{\mathrm{i}}\right)$, an electrostatic repulsion heuristic is applied to diversify the particles and to widely cover the search space [3]. This technique is used in an agent-based optimization algorithm for dynamic environments [5]. Therefore, this procedure is applied in each zone separately, hence each particle is considered as an electron. Then a force of $1 / r^{2}$ is applied, on the particles of each zone, until the maximum displacement of a particle during an iteration becomes lower than a given threshold $\epsilon$ (where $r$ is the distance between two particles, $\epsilon$ is typically equal to $10^{-4}$ ). At each iteration of this procedure, the particles are projected in the middle of the current zone, before reapplying the heuristic repulsion.

\section{Dynamic cluster topology (Dcluster)}

Dcluster is a dynamic topology that works as follows [1]. At each iteration, the particles are sorted in a list according to their personal best fitness in increasing order, so that the worst particle has an index equal to 1 in the list (the size of the list is equal to the size of the swarm). Then, the list is partitioned into several sub-lists which correspond to a cluster in the proposed topology. The first cluster which has the "worst" particle, called central cluster, is placed in the center of the topology. Each particle of the central cluster is connected to other clusters by one of their particles; the first worst particle of the central cluster is linked 
to the worst particle in the second cluster, the second one is linked to the third cluster also by its worst particle, and so on, as in Figure 1-(a). All clusters in this topology, including the central cluster, have a fully connected neighborhood. The reason why the central cluster is linked to each other cluster by only one gateway and with the worst particle of the latter is to avoid a premature convergence to a local optimum by slowing down the propagation of the information in the whole swarm. Figure 1-(b) illustrates the final structure of the proposed topology.

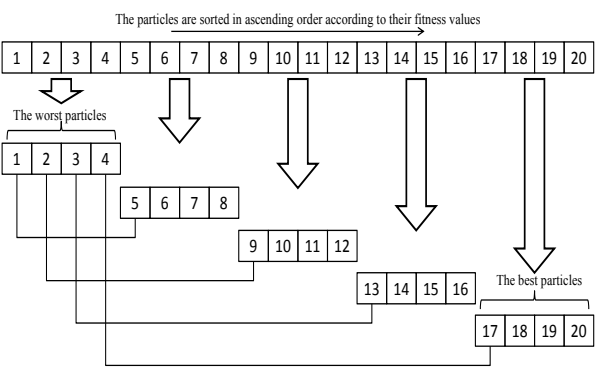

(a)

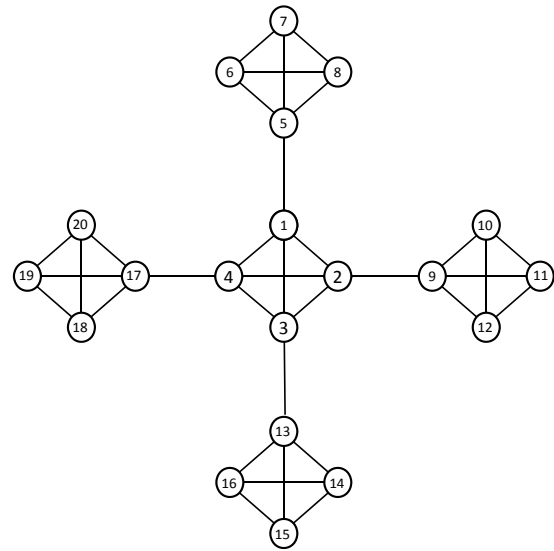

(b)

Fig. 1. (a) - The partitioning of the list into sub-lists. (b) - The structure of Dcluster topology.

\section{Experimental results}

Table 1 presents the settings of each problem, the number of function evaluations (Max. FEs), the success rate and the mean best value of 100 runs. The best results among those obtained by the two algorithms are shown in bold. From the experiments, we can notice that DPSO-2S (PSO-2S using Dcluster topology) obtains the best results on most of the functions used. Hence, DPSO-2S algorithm outperforms PSO-2S, except for Rosenbrock and Shifted Rosenbrock. Thus, this algorithm leads to a significant improvement over the previous PSO-2S.

\section{Conclusion}

In this paper, a new dynamic topology, called Dcluster, based on two static neighbourhood topologies was presented. Dcluster was integrated to our multiswarm algorithm PSO-2S. Experimental results indicate that Dcluster improves the search performance of the previous algorithm. 


\begin{tabular}{|c|c|c|c|c|c|c|c|}
\hline \multirow{2}{*}{ Function } & \multirow{2}{*}{$\begin{array}{l}\text { Search } \\
\text { space }\end{array}$} & \multirow{2}{*}{$\begin{array}{c}\text { Acceptable } \\
\text { error }\end{array}$} & \multirow{2}{*}{$\begin{array}{l}\text { Max. } \\
\text { FEs }\end{array}$} & \multicolumn{2}{|c|}{ DPSO-2S } & \multicolumn{2}{|c|}{ PSO-2S } \\
\hline & & & & $\begin{array}{c}\text { Mean } \\
\text { best }\end{array}$ & $\begin{array}{l}\text { Suc. } \\
\text { rate }\end{array}$ & $\begin{array}{c}\text { Mean } \\
\text { best }\end{array}$ & $\begin{array}{l}\text { Suc. } \\
\text { rate }\end{array}$ \\
\hline Rosenbrock & {$[-10,10]^{30}$} & & 40000 & $2.50 \mathrm{e}+001$ & $0.0 \%$ & $2.28 e_{+} 001$ & $0.0 \%$ \\
\hline Ackley & {$[-32,32]^{30}$} & 0.0001 & 40000 & $9.40 \mathrm{e}-003$ & $99 \%$ & $3.54 \mathrm{e}-001$ & $69 \%$ \\
\hline Griewank & {$[-100,100]^{30}$} & 0.0001 & 40000 & $2.19 \mathrm{e}-003$ & $78 \%$ & $3,88 \mathrm{e}-003$ & $72 \%$ \\
\hline Rastrigin & {$[-10,10]^{30}$} & 0.0001 & 40000 & $1.34 \mathrm{e}+000$ & $30 \%$ & $2.16 \mathrm{e}+000$ & $25 \%$ \\
\hline Sh. Rosenbrock & {$[-100,100]^{10}$} & 0.01 & 100000 & $5.25 \mathrm{e}+000$ & $5 \%$ & $5.98 \mathrm{e}-001$ & $75 \%$ \\
\hline Sh. Ackley & {$[-32,32]^{30}$} & 0.0001 & 100000 & $6.26 \mathrm{e}-002$ & $95 \%$ & $1.88 \mathrm{e}-001$ & $86 \%$ \\
\hline Sh. Griewank & {$[-600,600]^{30}$} & 0.0001 & 100000 & $5.16 \mathrm{e}-003$ & $66 \%$ & $6.11 \mathrm{e}-003$ & $61 \%$ \\
\hline Sh. Rastrigin & {$[-5,5]^{30}$} & 0.0001 & 100000 & $3.14 e_{+} 001$ & $0,0 \%$ & $5,36 \mathrm{e}+001$ & $0.0 \%$ \\
\hline
\end{tabular}

Table 1. Results of DPSO-2S using Dcluster topology and PSO-2S.

In conclusion, the improvement of the PSO-2S algorithm due to the integration of Dcluster topology opens the gate to apply this dynamic topology in other PSO algorithms.

\section{References}

1. El Dor A., Lemoine D., Clerc M., P. Siarry, L. Deroussi, and M. Gourgand. Dynamic Cluster in Particle Swarm Optimization algorithm. Natural Computing, October 2014. DOI: $10.1007 / \mathrm{s} 11047-014-9465-2$.

2. El Dor A., Clerc M., and Siarry P. A multi-swarm PSO using charged particles in a partitioned search space for continuous optimization. Computational Optimization and Applications, 53:271-295, 2012.

3. Conway J. and Sloane N. Sphere packings, lattices and groups. Springer (3rd ed.), 1999.

4. Kennedy J. A new optimizer using particle swarm theory. Proceedings of the Sixth International Symposium on Micro Machine and Human Science, pages 39-43, 1995.

5. Lepagnot J., Nakib A., Oulhadj H., and Siarry P. A new multiagent algorithm for dynamic continuous optimization. International Journal of Applied Metaheuristic Computing, 1(1):16-38, 2010.

6. J. Kennedy and R. Mendes. Population structure and particle swarm performance. In Proceedings of the 2002 IEEE Congress on Evolutionary Computation, CEC'02, pages 1671-1676, Honolulu, HI, USA, 2002.

7. R. Mendes, J. Kennedy, and J. Neves. The Fully Informed Particle Swarm: Simpler, Maybe Better. IEEE Trans. Evolutionary Computation, 8(3):204-210, June 2004. 